\title{
Methylation Analysis in Monozygotic Twins With Treatment-Resistant Schizophrenia and Discordant Responses to Clozapine
}

\begin{abstract}
Masataka Kikuchi ${ }^{1}$, Takanobu Nakazawa ${ }^{2}$, Makoto Kinoshita $^{3}$, Hidenaga Yamamori ${ }^{4,5,6}$, Yuka Yasuda ${ }^{4,7}$, Michiko Fujimoto ${ }^{4,5}$, Ryota Hashimoto ${ }^{4}$ and Shusuke Numata ${ }^{3 *}$

${ }^{1}$ Department of Genome Informatics, Graduate School of Medicine, Osaka University, Osaka, Japan, ${ }^{2}$ Department of Bioscience, Tokyo University of Agriculture, Tokyo, Japan, ${ }^{3}$ Department of Psychiatry, Institute of Biomedical Sciences, Tokushima University Graduate School, Tokushima, Japan, ${ }^{4}$ Department of Pathology of Mental Diseases, National Center of Neurology and Psychiatry, National Institute of Mental Health, Tokyo, Japan, ${ }^{5}$ Department of Psychiatry, Graduate School of Medicine, Osaka University, Osaka, Japan, ${ }^{6}$ Japan Community Health Care Organization Osaka Hospital, Osaka, Japan, ${ }^{7}$ Medical Corporation Foster, Osaka, Japan
\end{abstract}

OPEN ACCESS

Edited by: Hiroyuki Toda

National Defense Medical College, Japan

Reviewed by:

Tomoyo Sawada, Lieber Institute for Brain Development,

United States

Takumi Nakamura,

RIKEN Center for Brain Science

(CBS), Japan

*Correspondence:

Shusuke Numata

shu-numata@tokushima-u.ac.jp

Specialty section:

This article was submitted to Schizophrenia,

a section of the journal

Frontiers in Psychiatry

Received: 01 July 2021

Accepted: 24 August 2021

Published: 20 September 2021

Citation:

Kikuchi M, Nakazawa T, Kinoshita M, Yamamori $H$, Yasuda Y, Fujimoto M, Hashimoto $R$ and Numata S (2021)

Methylation Analysis in Monozygotic

Twins With Treatment-Resistant

Schizophrenia and Discordant

Responses to Clozapine.

Front. Psychiatry 12:734606.

doi: 10.3389/fpsyt.2021.734606
Schizophrenia is a mental illness that involves both genetic and environmental factors. Clozapine, an atypical antipsychotic, is a well-established therapy for treatmentresistant schizophrenia. In this study, we focused on a set of monozygotic twins with treatment-resistant schizophrenia in which one twin effectively responded to clozapine treatment and the other did not. Our previous study generated neurons from induced pluripotent stem (iPS) cells derived from these patients and compared the transcriptome profiles between mock- and clozapine-treated neurons. In this study, we performed genome-wide DNA methylation profiling to investigate the mechanisms underlying gene expression changes. First, we extracted the differentially methylated sites from each twin based on statistical analysis. Then, we combined the DNA methylation profiling with transcriptome profiling from our previous RNA-seq data. Among the genes with altered methylation and expression, we found the different proportions of the genes related to neuronal and synaptic functions between the clozapine responder and nonresponder (35.7 and 6.7\%, respectively). This trend was observed even when the basal differences between the responder and non-responder was excluded. These results suggest that effective clozapine action may correct the abnormalities of neuronal and

Keywords: schizophrenia, clozapine, DNA methylation, monozygotic twins, iPS cells

\section{INTRODUCTION}

Schizophrenia is characterized by positive symptoms, negative symptoms, and disturbances in basic cognitive functions. Its lifetime prevalence is $0.30-0.66 \%(1,2)$. Approximately $10-30 \%$ of patients with schizophrenia show little or no improvement in symptoms after multiple trials of monotherapy (3). Clozapine, which is an atypical antipsychotic drug, is considered for patients with such treatment-resistant schizophrenia. However, the molecular mechanism of action of clozapine is still not fully understood. Several issues should be addressed to uncover its mechanisms. One is the difficulty of obtaining brain tissues from living synapse functions in schizophrenia via changes in methylation. 
patients receiving clozapine. Another is the differences in the genetic backgrounds of patients. To overcome these challenges, we focused on a case of monozygotic twins. In this case, both of the identical twins exhibited treatment-resistant schizophrenia, but one twin responded well to clozapine treatment whereas the other twin did not. We had previously established induced pluripotent stem (iPS) cell lines from immortalized B cells from each patient and differentiated neurons from these iPS cells (4). We treated the iPS neurons from each twin with a mock treatment or clozapine, examined the genes expressed in response to clozapine by RNA-seq analysis, and identified differentially expressed genes (DEGs) specific to the clozapine responding twin. However, it remained unclear whether clozapine directly affects gene expression because changes in gene expression are closely related to epigenetic changes such as DNA methylation.

DNA methylation is an epigenetic change, that is, an acquired change in gene regulation that does not involve a change in DNA sequence. DNA methylation is more likely to occur at CpG sites, that is, $5^{\prime}$-CG-3' dinucleotide sequences, in the genome. DNA methylation is associated with transcriptional regulation, but the relationship is not necessarily the same for gene promoters and gene bodies. The hypermethylation of $\mathrm{CpG}$ sites in a promoter is negatively correlated with transcription (5). In contrast, DNA methylation is abundant in the bodies of actively transcribed genes (6-8). In other words, DNA methylation in gene bodies is positively correlated with transcription; therefore, its role is not related to gene silencing $(6,9,10)$. Aberrant DNA methylation has been reported in psychiatric disorders, including schizophrenia (11-16). Interestingly, a recent study found significant methylation changes in patients with schizophrenia, especially in treatment-resistant schizophrenia, reflecting the effects of clozapine (17). Additionally, several studies reported that clozapine altered DNA methylation levels in certain genes (18-21). This observation suggests that clozapine may act not only through direct modulation of gene expression but also through epigenetic changes.

In this study, we attempted to obtain a comprehensive DNA methylation profile of iPS neurons obtained from a pair of monozygotic twins with treatment-resistant schizophrenia and discordant responses to clozapine (one a responder and the other a non-responder), and we examined the changes in DNA methylation following clozapine treatment in each patient. Then, we investigated whether DNA methylation was involved in the differential expression of the genes identified in our previous RNA-seq data as exhibiting distinct responses to clozapine treatment.

\section{METHODS}

\section{Subjects}

Monozygotic twin patients with treatment-resistant schizophrenia were recruited at Osaka University Hospital. The details of the patients were previously described (4). The twins were 59-year-old Japanese females, both diagnosed with treatment-resistant schizophrenia. Each subject was diagnosed and assessed by at least two trained psychiatrists according to the Diagnostic and Statistical Manual of Mental Disorders, fourth edition (DSM-IV) criteria based on a structured clinical interview. Written informed consent was obtained from subjects after the procedures were fully explained.

\section{Generation of iPS Cells and in vitro Differentiation of iPS Cells Into Excitatory Neurons}

The generation of iPS cells from lymphoblastoid B-cell lines and in vitro differentiation of iPS cells into excitatory neurons were performed as previously described (4). In brief, immortalized lymphoblastoid B cells were electroporated with plasmids encoding hOCT3/4, hSOX2, hKLF4, hL-MYC, hLIN28 and dominant negative mTP53. After electroporation, cells were seeded onto SNL feeder cells and grown to form colonies. Subsequently, colonies similar to human ES cells were clonally isolated, morphologically selected and evaluated for the expression of pluripotency markers. For in vitro differentiation, iPS cells were transferred under feeder-free conditions onto Matrigel (Corning)-coated dishes and cultured in the chemically defined medium Essential 8 (Thermo Fisher Scientific) according to the manufacturer's instructions. The in vitro differentiation of iPS cells into neurons through neurogenin2 (Ngn2) overexpression was performed essentially according to Zhang et al. (22). On day 8, $1 \mu$ M clozapine (SigmaAldrich) or mock treatment was added to the culture medium. After 7 days, Ngn2-induced neurons were harvested for DNA isolation. The gene expression levels in iPS neurons measured by RNA-seq and related statistics were obtained from our previous study (4). In our previous study, total RNA was isolated from three clones obtained from each patient and equal amounts of total RNAs from each patient were combined and sequenced using the Illumina HiSeq2000 system (BGI, Beijing, China) (4). The reads were aligned to the human reference genome hg19. Identifying of DEGs between two samples was performed based on a Poisson distribution (BGI, Beijing, China). The calculated $p$-values were adjusted using FDR q-value to correct for multiple testing. Genes were identified as differentially expressed between the mock and clozapine treatments or between responder and non-responder at a significance level of FDR q-value $<0.05$.

\section{DNA Methylation Analysis}

Genomic DNA was extracted from neuronal nuclei using a Blood \& Cell Culture DNA kit (Qiagen). We used the same three clones of each twin as those analyzed by our previous RNA-seq analysis (4) for methylation analysis. Bisulfite conversion of $500 \mathrm{ng}$ of genomic DNA was performed with the EZ DNA methylation kit (Zymo Research). DNA methylation levels were assessed according to the manufacturer's instructions using Infinium ${ }^{\circledR}$ HumanMethylation450 BeadChips (Illumina Inc., San Diego, CA, USA), which enable the examination of DNA methylation status at $485,577 \mathrm{CpG}$ sites, and the resulting data were analyzed using the methylation analysis module within the BeadStudio software (Illumina Inc.). The data were mapped to the hg19 genome. The regions described as TSS200 and TSS1500 in this annotation file were defined as the promoter regions. For 
methylation analysis, IDAT files were processed using the $\mathrm{R}$ software package minfi (23). The raw signal data were normalized by the preprocessQuantile function. The methylation status of each $\mathrm{CpG}$ site was represented as a $\beta$-value that ranged from 0 (completely unmethylated) to 1 (fully methylated). We analyzed 341,629 sites that satisfied the following criteria: (1) $\beta$-values with detection $p$-values $<0.01$; (2) CpGs with probes having $<3$ beads; (3) no probe single nucleotide polymorphisms (SNPs) with minor allele frequencies (MAFs) $\geq 1 \%$ in the HapMapJPT population; (4) no probe cross-reactivity; and (5) no SNPs at $\mathrm{CpG}$ sites and single-base extension sites. A list of probes and their corresponding MAF values in the Japanese population was derived from Okamura et al. (24). We excluded sites with SNPs and in cross-reactive regions based on a list derived from Chen et al. (25). We performed dmpFinder function in the minfi package to identify positions that were differentially methylated. Methylation sites were identified as differentially methylated between the mock and clozapine treatments or between responder and non-responder at a significance level of $p$-value $<0.01$. The calculated $p$-values were adjusted using FDR q-value to correct for multiple testing, although there were no methylation sites differentially methylated between the mock and clozapine treatments at a significance level of FDR q-value $<0.05$.

\section{The Direction of Change for the mRNA Expression Level and the DNA Methylation Level of the DEGs}

The same direction means that both the mRNA expression level and the DNA methylation level are higher or lower in clozapine compared to mock treatment. The opposite direction indicates highly express and lower methylate in clozapine compared to mock treatment, or vice versa.

\section{Genes Associated With Neuronal and Synaptic Functions}

Genes associated with neuronal and synaptic functions were defined as those genes annotated with Gene Ontology terms including the following words: "neuro," "synapse," or "synaptic." We used the information from the Gene Ontology project (http:// geneontology.org/) and R software package GO.db. We obtained 2,756 genes after the procedures. Furthermore, we selected 2,399 genes overlapped with 15,466 genes that we treated in the RNAseq and DNA methylation analyses.

\section{Gene Functional Enrichment Analysis}

Gene functional enrichment analysis was performed using Metascape software (26). Metascape reports a term that an input gene list overrepresented as an enrichment cluster and prevents redundancies in terms across different ontology sources.

\section{Statistical Analysis}

To examine the extent of overlap between the DEGs and the genes related to neuronal and synaptic functions, we calculated the $p$-value by hypergeometric distribution test and the expected numbers as follows:

$$
\begin{gathered}
P(X=x)=\frac{\left(\frac{m}{x}\right)\left(\frac{N-m}{n-x}\right)}{\left(\frac{N}{n}\right)}, \\
\text { Expected }=\frac{m n}{N},
\end{gathered}
$$

where $x$ is the number of genes that overlapped between the DEGs and the genes related to neuronal and synaptic functions, and $m$ and $n$ are the numbers of the DEGs and the genes related to neuronal and synaptic functions (2,399 genes), respectively. $N$ is the total number of genes $(15,466$ genes that we treated in the RNA-seq and DNA methylation analyses).

\section{RESULTS}

\section{Changes in DNA Methylation Following Clozapine Treatment in iPS Neurons From Twins With Discordant Responses to Clozapine}

We show an overview of the present study in Figure 1. We performed genome-wide DNA methylation profiling using differentiated neurons from iPS cell clones obtained from clozapine responders and non-responders. We analyzed 341,629 of 485,577 methylation sites based on robustness criteria (see section Methods). To identify positions that were differentially methylated following clozapine treatment, we compared the DNA methylation levels of each methylation site between the mock and clozapine-treated neurons in each twin. We extracted the changed methylation sites from each comparison at a significance level of $p$-value $<0.01$. As a result, we identified 2,229 and 1,384 methylation sites in the clozapine responder and non-responder, respectively (Supplementary Tables 1, 2). Among these changed methylation sites, only 21 sites were overlapped between the twins. After we excluded the 21 overlapped sites from the changed methylation sites in each twin, these patient-specific methylation sites were involved 1,789 and 1,145 genes in the clozapine responder and nonresponder, respectively. We used these methylation data in the subsequent analyses.

\section{Combined Transcriptome Profiling With DNA Methylation Profiling}

We recently identified DEGs following clozapine treatment in iPS neurons from the same twins by performing RNA-seq analysis (4). We found 714 DEGs specific to the clozapine responder and 277 DEGs specific to the clozapine non-responder (FDR q-value $<0.05)$. Among 714 DEGs identified in the clozapine responder, 84 genes were located near the responder-specific methylation sites (Supplementary Table 3). Of the 84 DEGs, 17 DEGs, including MECP2 (methyl-CpG-binding protein 2), which has been implicated in schizophrenia and autism as well as Rett syndrome $(27,28)$, differed in the opposite direction of the change in the methylation sites in the promoter regions 


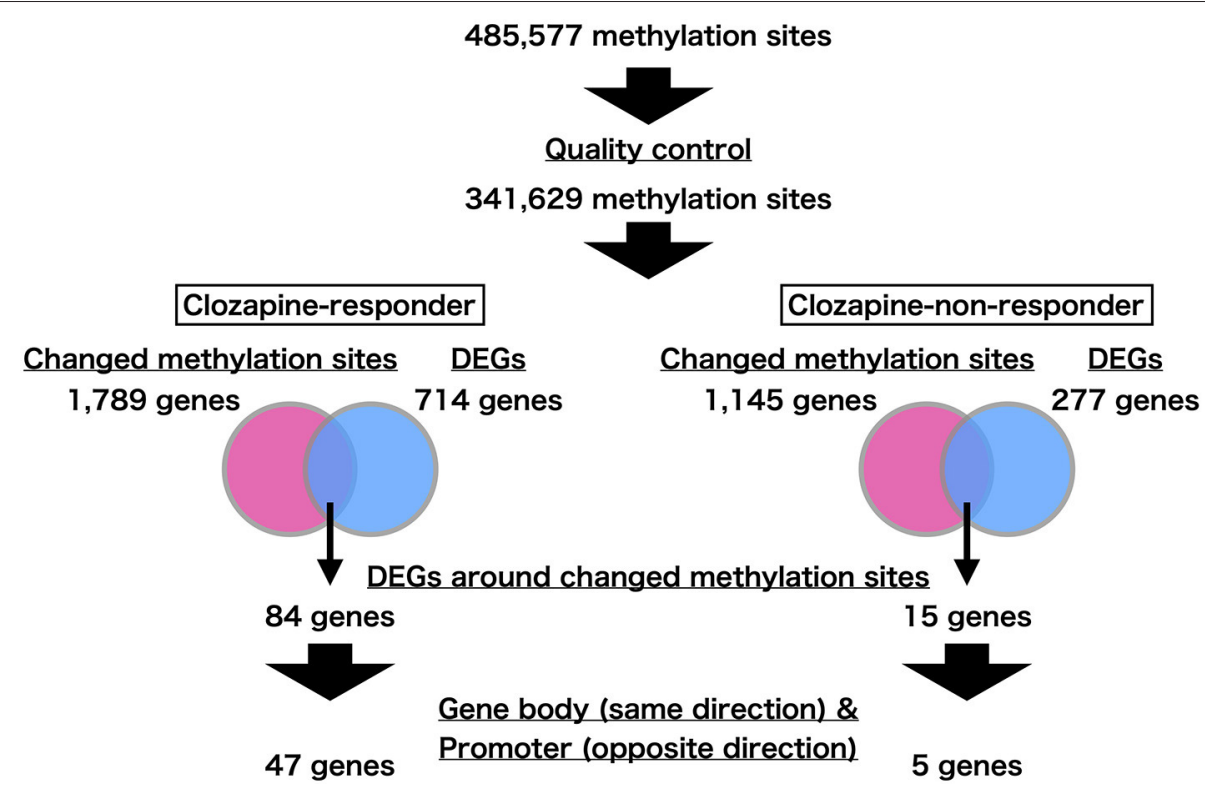

FIGURE 1 | Flowchart of the present study. We first found the genes near the changed methylation sites. We next extracted differentially expressed genes (DEGs) from this set based on transcriptome profiling. Focusing on the methylation sites in gene bodies and promoter regions, we identified genes whose expression changes were consistent with the methylation changes.

(Table 1; Supplementary Figure 1), and 32 DEGs showed the same direction of change for the mRNA expression level and the DNA methylation level in the corresponding gene body (Table 2). Of the DEGs associated with the promoters and gene bodies, 2 genes [STOX2 (storkhead box 2) and TBC1D16 (TBC1 domain family member 16)] were common. Among 277 DEGs identified in the clozapine non-responder, 15 genes were located near the non-responder-specific methylation sites (Supplementary Table 4). Of these 15 DEGs, 2 DEGs differed in the opposite direction of the change in the methylation sites in the promoter regions (Table 1), while 3 DEGs showed the same direction of change in the mRNA expression level and the DNA methylation level in the corresponding gene body (Table 2).

Then, we examined the proportion of genes associated with neuronal and synaptic functions (Table 3). Among the genes near the changed methylation sites, 300 of the 1,789 genes (16.8\%) in the clozapine responder and 190 of the 1,145 genes $(16.6 \%)$ in the clozapine non-responder were associated with neuronal and synaptic functions. Among the genes with altered methylation and expression, 30 of the 84 genes $(35.7 \%)$ in the clozapine responder and 1 of the 15 genes $(6.7 \%)$ in the clozapine non-responder were associated with neuronal and synaptic functions. The number of the genes with neuronal and synaptic functions in the clozapine responder was significantly 2.3 times higher than the expected value $(p$-value $=2.96 \mathrm{E}-06$; \#observed genes / \#expected genes $=2.30$ ), whereas the number of the genes in non-responder was lower than the expected value $(p$-value $=0.220$; observed genes $/$ \#expected genes $=$ 0.430 ). When we performed gene functional enrichment analysis to investigate the functions of these 84 DEGs, we found axon guidance (FDR q-value $=1.59 \mathrm{E}-07$ ) and neuron projection morphogenesis $($ FDR q-value $=1.21 \mathrm{E}-05)$ in the gene functional enrichment clusters (Supplementary Figure 2). In contrast, we did not find any statistically significantly enriched function in the clozapine non-responder. Among the genes whose expression changes were consistent with the methylation changes in the gene body or promoter regions, 19 of the 47 genes (40.4\%) in the clozapine responder were significantly associated with neuronal and synaptic functions ( $p$-value $=2.53 \mathrm{E}-05$; \#observed genes / \# expected genes $=2.61$ ), while there were no associations in the clozapine non-responder $(p$-value $=0.430$; \#observed genes / \# expected genes $=0.00$ ).

\section{The Differences of the Basal Methylation States of the Responder and Non-responder}

We finally examined the differences of the basal methylation states of the responder and non-responder. We identified 3,065 and 2,980 methylation sites that changed between the twins in the mock and clozapine treatments at a significance level of $p$ value $<0.01$, respectively. Among these changed methylation sites, 308 sites were overlapped between the two treatments. Each changed methylation sites were involved 2,305 and 2,241 genes in the mock and clozapine treatments, respectively. The RNA-seq analysis found 1,705 DEGs in the mock treatment and 806 DEGs in the clozapine treatment (FDR q-value $<0.05$ ). Among 1,705 DEGs identified in mock treatment, 133 genes were located near the 3,065 changed methylation sites. Among 806 DEGs identified in the clozapine treatment, 88 genes were located near the 2,980 changed methylation sites. The 133 DEGs in mock treatment were overlapped with only 5 genes among the 84 DEGs response 
TABLE 1 | Genes changed in the opposite direction to promoter CpG sites in response to clozapine.

\begin{tabular}{|c|c|c|c|c|c|c|}
\hline \multirow[b]{2}{*}{ CpG probe } & \multirow[b]{2}{*}{ Gene } & \multirow[b]{2}{*}{ Description } & \multicolumn{2}{|c|}{ Responder } & \multicolumn{2}{|c|}{ Non-responder } \\
\hline & & & $\begin{array}{l}\text { Log2 ratio of } \\
\text { methylation }\end{array}$ & $\begin{array}{l}\text { Log2 ratio of } \\
\text { mRNA }\end{array}$ & $\begin{array}{l}\text { Log2 ratio of } \\
\text { methylation }\end{array}$ & $\begin{array}{l}\text { Log2 ratio of } \\
\text { mRNA }\end{array}$ \\
\hline \multicolumn{7}{|l|}{ Responder } \\
\hline cg02025573 & KIAA0100 & KIAA0100 & 0.157 & -0.260 & 0.023 & -0.032 \\
\hline cg06012574 & ANKRD11 & ankyrin repeat domain 11 & 0.239 & -0.285 & -0.009 & 0.010 \\
\hline cg06502510 & ITSN1 & intersectin 1 & 0.276 & -0.368 & -0.246 & 0.030 \\
\hline $\operatorname{cg} 08463298$ & EIF4G3 & $\begin{array}{l}\text { eukaryotic translation initiation factor } 4 \text { gamma } \\
3\end{array}$ & 0.101 & -0.239 & -0.082 & 0.030 \\
\hline cg10232470 & TBC1D16 & TBC1 domain family member 16 & 0.389 & -0.324 & 0.001 & -0.073 \\
\hline cg12989020 & ARID1B & AT-rich interaction domain 1B & 0.171 & -0.252 & 0.120 & 0.016 \\
\hline cg13189687 & MECP2 & methyl-CpG binding protein 2 & 0.047 & -0.341 & -0.001 & 0.070 \\
\hline cg16010370 & TKT & transketolase & -0.303 & 0.151 & 0.078 & -0.018 \\
\hline cg18165031 & $\mathrm{ERH}$ & enhancer of rudimentary homolog & -0.456 & 0.266 & -0.195 & 0.062 \\
\hline cg20016416 & SPTBN2 & spectrin beta, non-erythrocytic 2 & 0.007 & -0.272 & 0.010 & -0.043 \\
\hline cg20465219 & PSMA5 & proteasome subunit alpha 5 & -0.207 & 0.312 & -0.001 & -0.064 \\
\hline cg20715295 & PAFAH1B3 & $\begin{array}{l}\text { platelet activating factor acetylhydrolase } 1 \mathrm{~b} \\
\text { catalytic subunit } 3\end{array}$ & -0.064 & 0.155 & -0.004 & 0.068 \\
\hline cg24798305 & SMC1A & structural maintenance of chromosomes $1 \mathrm{~A}$ & 0.034 & -0.244 & -0.002 & 0.089 \\
\hline $\operatorname{cg} 25419628$ & NHLRC2 & NHL repeat containing 2 & 0.223 & -1.077 & -0.190 & 0.350 \\
\hline cg25669309 & STOX2 & storkhead box 2 & 0.734 & -0.844 & -0.316 & -0.016 \\
\hline $\operatorname{cg} 27489994$ & TPT1 & tumor protein, translationally-controlled 1 & -0.238 & 0.101 & -0.091 & 0.062 \\
\hline cg27663249 & SRRM2 & serine/arginine repetitive matrix 2 & 0.020 & -0.221 & 0.022 & -0.072 \\
\hline \multicolumn{7}{|c|}{ Non-responder } \\
\hline cg00828305 & HNRNPA3 & heterogeneous nuclear ribonucleoprotein A3 & 0.063 & 0.026 & -0.222 & 0.167 \\
\hline cg19561607 & BCAT1 & branched chain amino acid transaminase 1 & -0.029 & -0.030 & -0.020 & 0.202 \\
\hline
\end{tabular}

Log2 ratio was calculated as the mean value in clozapine treatment divided by the mean value in mock treatment.

to clozapine in the clozapine responder. Additionally, there were no genes overlapped between 133 DEGs in mock treatment and 15 DEGs in the clozapine non-responder.

\section{DISCUSSION}

We examined the DNA methylation changes following clozapine treatment in iPS neurons from a pair of twins with discordant responses to clozapine. To date, there have been several studies of schizophrenia using human iPS cells (29). Applying iPS cell-based technology may provide new insight into the therapeutic mechanism of clozapine. We demonstrated that there were only a small number of $\mathrm{CpG}$ sites that were common between the clozapine responder and non-responder among the changed methylation sites. Then, we combined our previous transcriptome profiling data with DNA methylation profiling to identify genes whose expression changes and methylation changes were consistent. When we examined the correlations between the mRNA expression and DNA methylation levels, we found cases with changes in not only the opposite direction of the promoter but also the same direction in the corresponding gene body. These findings were in accord with recent reports that the bodies of actively transcribed genes are enriched in DNA methylation (6-8). There are two hypotheses about the function of DNA methylation in gene bodies (30). One is that it facilitates transcription elongation and/or co-transcriptional splicing. The other is that it represses intragenic cryptic promoters. Importantly, we demonstrated that genes related to neuronal and synaptic functions were observed at a higher frequency among the genes with altered DNA methylation and expression in the clozapine responder than in the clozapine non-responder. These results suggest that effective clozapine action may normalize the abnormalities of neuronal and synaptic functions via methylation changes.

We revealed increased DNA methylation in the promoter region and decreased expression in the MECP2 gene following clozapine treatment in the clozapine responder (Supplementary Figure 1). Basic research has shown that MeCP2 plays an important role in mediating synaptic transmission in the CNS $(31,32)$, and mutations in MECP2 are well-known to be implicated in neurodevelopmental disorders, including schizophrenia $(28,33)$. MECP2 binds to the promoter regions of methylated genes and suppresses their expression $(34,35)$. It was reported that MECP2 regulates thousands of genes in the hypothalamus and that $85 \%$ of these target genes were transcriptionally activated by MECP2 using comprehensive mRNA expression analysis of MECP2 knockout and MECP2 overexpression mice (36). Our results suggest that clozapine 
TABLE 2 | Genes changed in the same direction as the gene body CpG sites in response to clozapine.

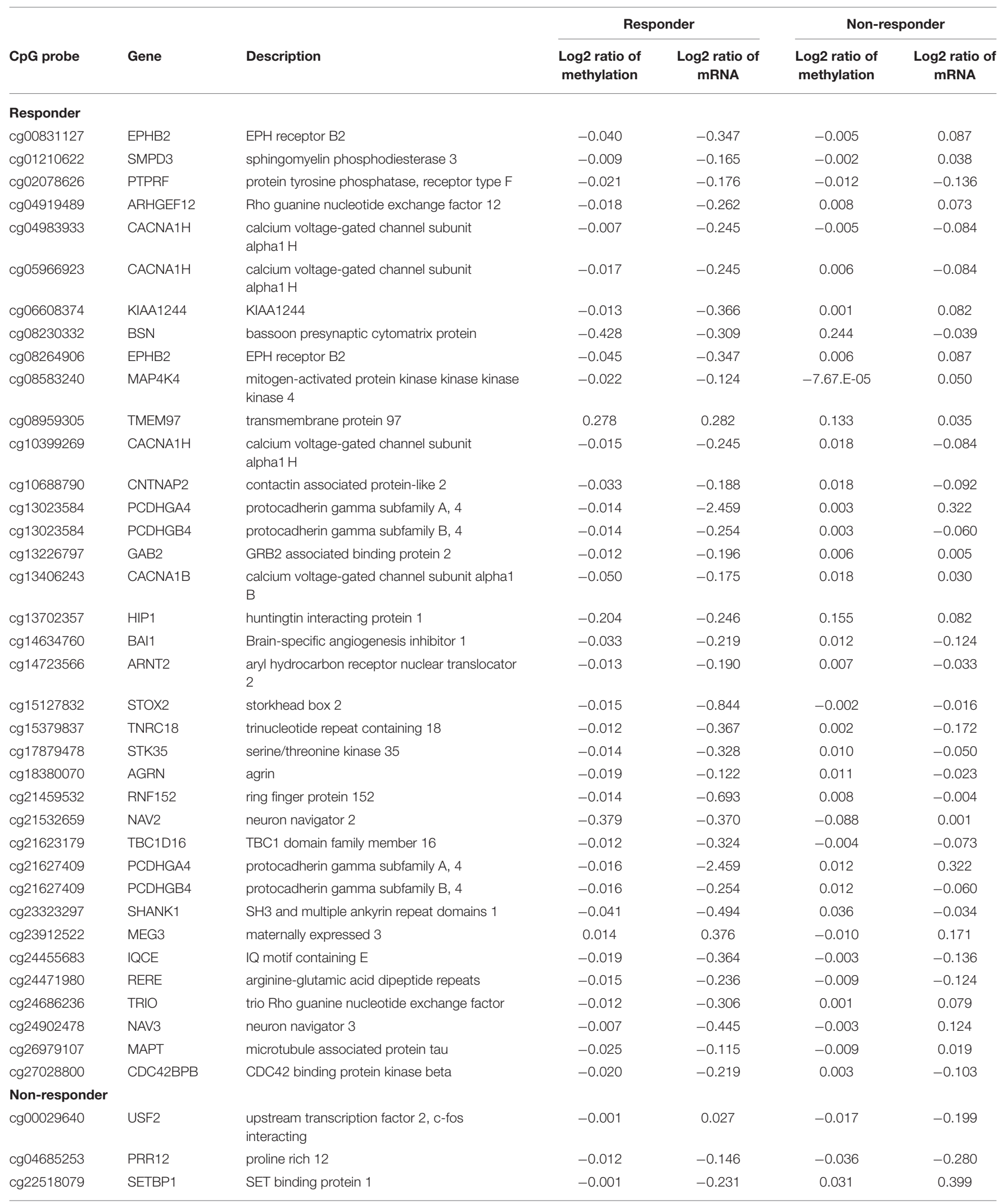

Log2 ratio was calculated as the mean value in clozapine treatment divided by the mean value in mock treatment. 
TABLE 3 | The proportion of genes associated with neuronal and synaptic functions.

\begin{tabular}{|c|c|c|c|c|c|}
\hline & Neuronal genes (observed) & Corresponding genes & Expected & Observed/expected & $P$-value \\
\hline \multicolumn{6}{|l|}{ Responder } \\
\hline Changed methylation sites & $300(16.8 \%)$ & 1,789 & 277.5 & 1.081 & 0.008 \\
\hline DEGs around changed methylation sites & 30 (35.7\%) & 84 & 13 & 2.302 & 2.96.E-06 \\
\hline Gene body (same direction) and Promoter (opposite direction) & $19(40.4 \%)$ & 47 & 7.3 & 2.606 & 2.53.E-05 \\
\hline \multicolumn{6}{|l|}{ Non-responder } \\
\hline Changed methylation sites & $190(16.6 \%)$ & 1,145 & 177.6 & 1.07 & 0.019 \\
\hline DEGs around changed methylation sites & $1(6.7 \%)$ & 15 & 2.3 & 0.43 & 0.22 \\
\hline Gene body (same direction) and Promoter (opposite direction) & $0(0.0 \%)$ & 5 & 0.8 & 0 & 0.43 \\
\hline
\end{tabular}

$P$-values were calculated by hypergeometric distribution test.

may affect the mRNA expression level of the MECP2 gene via DNA methylation as well as the transcriptomes of multiple downstream genes.

Among the methylation sites that specifically responded to clozapine in the responder, cg03942932 showed significant methylation changes in a previous meta-analysis study of DNA methylation changes in schizophrenia and controls using blood samples (17). In addition, the responder-specific cg19939130 was also significantly altered between schizophrenia and controls in a study using African American blood samples (14). Interestingly, DOT1L (DOT1 like histone lysine methyltransferase), which was a gene with methylation sites that varied specifically in responders, was reported as the gene with the most variable methylation sites in blood between treatment-resistant and non-treatment-resistant schizophrenic patients (17).

In our previous study, we performed whole exome sequencing analysis of the twins and did not observe any genomic discordance on exons between the twins (4). However, there may be differences in mutations in non-coding regulatory regions such as promoters between the twins, and such mutations may affect methylation. Detailed observations by targeted sequencing will be necessary. If there is no genomic discordance between the twins, the reason for their discordance in clozapine response may be somatic changes during development. We also cannot exclude the possibilities that the differences in clozapine responsiveness observed between the twins' iPS cell-derived neurons are affected by the reprogramming process that can largely reset DNA methylation patterns in somatic cells and/or by the NGN2-memdiated neuronal differentiation. Further studies with neurons directly differentiated from the twins' somatic cells or iPS cell-derived neurons differentiated without viral overexpression of NGN2 would be necessary to validate our findings.

There are several limitations in the present study. As this is a case report with a pair of monozygotic twins, the findings need to be validated in studies with larger cohort including healthy control individuals to demonstrate the disease relevancy. In addition, further in vivo experiments and experiments with animal model will be required to reveal how the DNA methylation levels of the $\mathrm{CpG}$ sites and mRNA expression levels of the corresponding genes are altered by clozapine in the responder-derived neurons and what determines the responsiveness to clozapine.

\section{DATA AVAILABILITY STATEMENT}

The original contributions presented in the study are included in the article/Supplementary Material, further inquiries can be directed to the corresponding author/s.

\section{ETHICS STATEMENT}

The studies involving human participants were reviewed and approved by The Research Ethical Committees of Osaka University and Tokushima University. The patients/participants provided their written informed consent to participate in this study.

\section{AUTHOR CONTRIBUTIONS}

MKik and SN contributed to the study design and wrote the manuscript. MKik analyzed the data. TN performed the iPS cell experiments. MKin obtained the DNA methylation data. HY, YY, and MF contributed to the data collection. MKik, TN, MKin, HY, YY, MF, RH, and SN contributed text to the manuscript. All authors read and approved the final manuscript.

\section{FUNDING}

This work was supported by Grants-in-Aid for Scientific Research [grant numbers 17K15049 (MKik), 20K15778 (MKik), 21H02628 (TN), $21 \mathrm{H} 00213$ (TN), $20 \mathrm{~K} 07945$ (MF), JP20H03611 $(\mathrm{RH})$, and JP18KT0022 (RH)] from the Ministry of Education, Culture, Sports, Science and Technology (MEXT); the Health and Labour Sciences Research Grants for Comprehensive Research on Persons with Disabilities [16dk0307065 h001 (TN, MKik, SN, and RH)], JP21gm1310003 (TN), JP21dk0307103 (RH) from the Japan Agency for Medical Research and Development (AMED); a grant from the Takeda Science Foundation (TN); a grant from the Asahi Glass Foundation (TN); a grant from 
the Naito Foundation (TN); and an Intramural Research Grant (3-1) for Neurological and Psychiatric Disorders of NCNP (RH). The funders had no role in the study design, data collection and analysis, decision to publish, or preparation of the manuscript.

\section{REFERENCES}

1. Uno Y, Coyle JT. Glutamate hypothesis in schizophrenia. Psychiatry Clin Neurosci. (2019) 73:204-15. doi: 10.1111/pcn.12823

2. van Os J, Kapur S. Schizophrenia. Lancet. (2009) 374:635-45. doi: 10.1016/S0140-6736(09)60995-8

3. Lehman AF, Lieberman JA, Dixon LB, McGlashan TH, Miller AL, Perkins DO, et al. Practice guideline for the treatment of patients with schizophrenia, second edition. Am J Psychiatry. (2004) 161 (2 Suppl.):1-56.

4. Nakazawa T, Kikuchi M, Ishikawa M, Yamamori H, Nagayasu K, Matsumoto $\mathrm{T}$, et al. Differential gene expression profiles in neurons generated from lymphoblastoid B-cell line-derived iPS cells from monozygotic twin cases with treatment-resistant schizophrenia and discordant responses to clozapine. Schizophr Res. (2017) 181:75-82. doi: 10.1016/j.schres.2016.10.012

5. Weber M, Hellmann I, Stadler MB, Ramos L, Pääbo S, Rebhan M, et al. Distribution, silencing potential and evolutionary impact of promoter DNA methylation in the human genome. Nat Genet. (2007) 39:457-66. doi: $10.1038 /$ ng 1990

6. Lister R, Pelizzola M, Dowen RH, Hawkins RD, Hon G, Tonti-Filippini J, et al. Human DNA methylomes at base resolution show widespread epigenomic differences. Nature. (2009) 462:315-22. doi: 10.1038/nature08514

7. Zemach A, McDaniel IE, Silva P, Zilberman D. Genome-wide evolutionary analysis of eukaryotic DNA methylation. Science. (2010) 328:916-9. doi: $10.1126 /$ science.1186366

8. Feng S, Cokus SJ, Zhang X, Chen PY, Bostick M, Goll MG, et al. Conservation and divergence of methylation patterning in plants and animals. Proc Natl Acad Sci USA. (2010) 107:8689-94. doi: 10.1073/pnas.1002720107

9. Bender CM, Gonzalgo ML, Gonzales FA, Nguyen CT, Robertson KD, Jones PA. Roles of cell division and gene transcription in the methylation of CpG islands. Mol Cell Biol. (1999) 19:6690-8. doi: 10.1128/MCB.19.10.6690

10. Varley KE, Gertz J, Bowling KM, Parker SL, Reddy TE, Pauli-Behn F, et al. Dynamic DNA methylation across diverse human cell lines and tissues. Genome Res. (2013) 23:555-67. doi: 10.1101/gr.147942.112

11. Chen J, Zang Z, Braun U, Schwarz K, Harneit A, Kremer T, et al. Association of a reproducible epigenetic risk profile for schizophrenia with brain methylation and function. JAMA Psychiatry. (2020) 77:628-36. doi: 10.1001/jamapsychiatry.2019.4792

12. Huang HS, Matevossian A, Whittle C, Kim SY, Schumacher A, Baker SP, et al. Prefrontal dysfunction in schizophrenia involves mixed-lineage leukemia 1regulated histone methylation at GABAergic gene promoters. J Neurosci. (2007) 27:11254-62. doi: 10.1523/JNEUROSCI.3272-07.2007

13. Kinoshita M, Numata S, Tajima A, Shimodera S, Ono S, Imamura A, et al. DNA methylation signatures of peripheral leukocytes in schizophrenia. Neuromolecular Med. (2013) 15:95-101. doi: 10.1007/s12017-012-8198-6

14. Montano C, Taub MA, Jaffe A, Briem E, Feinberg JI, Trygvadottir R, et al. Association of DNA methylation differences with schizophrenia in an epigenome-wide association study. JAMA Psychiatry. (2016) 73:506-14. doi: 10.1001/jamapsychiatry.2016.0144

15. Numata S, Ye T, Herman M, Lipska BK. DNA methylation changes in the postmortem dorsolateral prefrontal cortex of patients with schizophrenia. Front Genet. (2014) 5:280. doi: 10.3389/fgene.2014.00280

16. Wockner LF, Noble EP, Lawford BR, Young RM, Morris CP, Whitehall VL, et al. Genome-wide DNA methylation analysis of human brain tissue from schizophrenia patients. Transl Psychiatry. (2014) 4:e339. doi: 10.1038/tp.2013.111

17. Hannon E, Dempster EL, Mansell G, Burrage J, Bass N, Bohlken MM, et al. DNA methylation meta-analysis reveals cellular alterations in psychosis and markers of treatment-resistant schizophrenia. Elife. (2021) 10:e58430. doi: 10.7554/eLife.58430

\section{SUPPLEMENTARY MATERIAL}

The Supplementary Material for this article can be found online at: https://www.frontiersin.org/articles/10.3389/fpsyt. 2021.734606/full\#supplementary-material

18. Dong E, Nelson M, Grayson DR, Costa E, Guidotti A. Clozapine and sulpiride but not haloperidol or olanzapine activate brain DNA demethylation. Proc Natl Acad Sci USA. (2008) 105:13614-9. doi: 10.1073/pnas.0805493105

19. Guidotti A, Grayson DR. DNA methylation and demethylation as targets for antipsychotic therapy. Dialogues Clin Neurosci. (2014) 16:419-29. doi: 10.31887/DCNS.2014.16.3/aguidotti

20. Dong E, Tueting P, Matrisciano F, Grayson DR, Guidotti A. Behavioral and molecular neuroepigenetic alterations in prenatally stressed mice: relevance for the study of chromatin remodeling properties of antipsychotic drugs. Transl Psychiatry. (2016) 6:e711. doi: 10.1038/tp.2015.191

21. Kinoshita M, Numata S, Tajima A, Yamamori H, Yasuda Y, Fujimoto M, et al. Effect of clozapine on DNA methylation in peripheral leukocytes from patients with treatment-resistant schizophrenia. Int J Mol Sci. (2017) 18:632. doi: 10.3390/ijms18030632

22. Zhang Y, Pak C, Han Y, Ahlenius H, Zhang Z, Chanda S, et al. Rapid singlestep induction of functional neurons from human pluripotent stem cells. Neuron. (2013) 78:785-98. doi: 10.1016/j.neuron.2013.05.029

23. Aryee MJ, Jaffe AE, Corrada-Bravo H, Ladd-Acosta C, Feinberg AP, Hansen $\mathrm{KD}$, et al. Minfi: a flexible and comprehensive bioconductor package for the analysis of infinium DNA methylation microarrays. Bioinformatics. (2014) 30:1363-9. doi: 10.1093/bioinformatics/btu049

24. Okamura K, Kawai T, Hata K, Nakabayashi K. Lists of HumanMethylation450 BeadChip probes with nucleotide-variant information obtained from the phase 3 data of the 1000 genomes project. Genom Data. (2016) 7:67-9. doi: 10.1016/j.gdata.2015.11.023

25. Chen YA, Lemire M, Choufani S, Butcher DT, Grafodatskaya D, Zanke BW, et al. Discovery of cross-reactive probes and polymorphic CpGs in the Illumina Infinium HumanMethylation450 microarray. Epigenetics. (2013) 8:203-9. doi: 10.4161/epi.23470

26. Zhou Y, Zhou B, Pache L, Chang M, Khodabakhshi AH, Tanaseichuk O, et al. Metascape provides a biologist-oriented resource for the analysis of systemslevel datasets. Nat Commun. (2019) 10:1523. doi: 10.1038/s41467-01909234-6

27. Nishiyama J. Plasticity of dendritic spines: molecular function and dysfunction in neurodevelopmental disorders. Psychiatry Clin Neurosci. (2019) 73:541-50. doi: 10.1111/pcn.12899

28. Chin EWM, Goh ELK. MeCP2 dysfunction in rett syndrome and neuropsychiatric disorders. Methods Mol Biol. (2019) 2011:573-91. doi: 10.1007/978-1-4939-9554-7_33

29. Shen X, Yeung HT, Lai KO. Application of human-induced pluripotent stem cells (hiPSCs) to study synaptopathy of neurodevelopmental disorders. Dev Neurobiol. (2019) 79:20-35. doi: 10.1002/dneu.22644

30. Greenberg MVC, Bourc'his D. The diverse roles of DNA methylation in mammalian development and disease. Nat Rev Mol Cell Biol. (2019) 20:590 607. doi: 10.1038/s41580-019-0159-6

31. Fukuda T, Itoh M, Ichikawa T, Washiyama K, Goto Y. Delayed maturation of neuronal architecture and synaptogenesis in cerebral cortex of Mecp2-deficient mice. J Neuropathol Exp Neurol. (2005) 64:537-44. doi: $10.1093 /$ jnen/64.6.537

32. Na ES, Monteggia LM. The role of MeCP2 in CNS development and function. Horm Behav. (2011) 59:364-8. doi: 10.1016/j.yhbeh.2010.05.014

33. Chen $\mathrm{CH}$, Cheng MC, Huang A, Hu TM, Ping LY, Chang YS. Detection of rare methyl-CpG binding protein 2 gene missense mutations in patients with schizophrenia. Front Genet. (2020) 11:476. doi: 10.3389/fgene.2020. 00476

34. Lewis JD, Meehan RR, Henzel WJ, Maurer-Fogy I, Jeppesen P, Klein $\mathrm{F}$, et al. Purification, sequence, and cellular localization of a novel chromosomal protein that binds to methylated DNA. Cell. (1992) 69:905-14. doi: 10.1016/0092-8674(92)90610-O 
35. Nan X, Campoy FJ, Bird A. MeCP2 is a transcriptional repressor with abundant binding sites in genomic chromatin. Cell. (1997) 88:471-81. doi: 10.1016/S0092-8674(00)81887-5

36. Chahrour M, Jung SY, Shaw C, Zhou X, Wong ST, Qin J, et al. $\mathrm{MeCP} 2$, a key contributor to neurological disease, activates and represses transcription. Science. (2008) 320:1224-9. doi: 10.1126/science. 1153252

\section{Conflict of Interest: YY is employed by Medical Corporation Foster.}

The remaining authors declare that the research was conducted in the absence of any commercial or financial relationships that could be construed as a potential conflict of interest.
Publisher's Note: All claims expressed in this article are solely those of the authors and do not necessarily represent those of their affiliated organizations, or those of the publisher, the editors and the reviewers. Any product that may be evaluated in this article, or claim that may be made by its manufacturer, is not guaranteed or endorsed by the publisher.

Copyright (C) 2021 Kikuchi, Nakazawa, Kinoshita, Yamamori, Yasuda, Fujimoto, Hashimoto and Numata. This is an open-access article distributed under the terms of the Creative Commons Attribution License (CC BY). The use, distribution or reproduction in other forums is permitted, provided the original author(s) and the copyright owner(s) are credited and that the original publication in this journal is cited, in accordance with accepted academic practice. No use, distribution or reproduction is permitted which does not comply with these terms. 